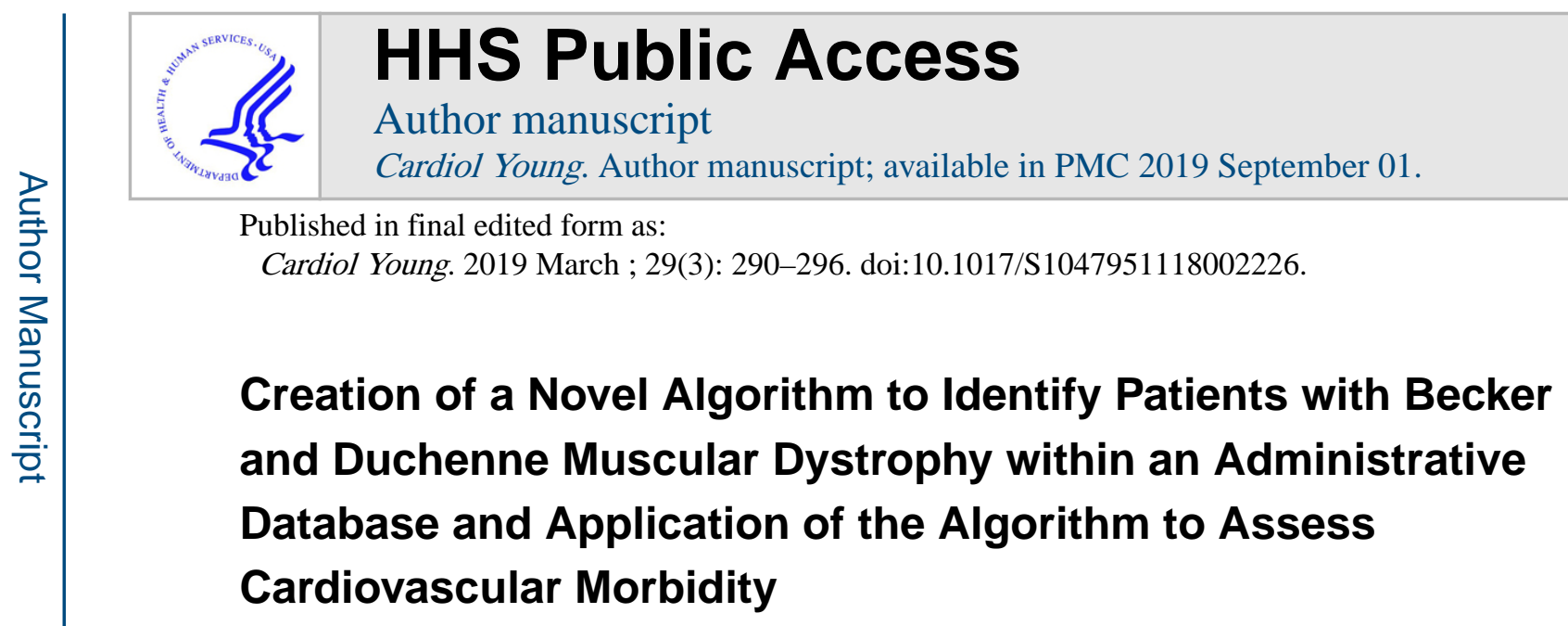

\author{
Jonathan H Soslow, M.D., M.S.C.I. ${ }^{a}$ [Assistant Professor], Matthew Hall ${ }^{b}$, W Bryan \\ Burnette $^{c}$, Kan Hor ${ }^{d}$, Joanne Chisolm ${ }^{d}$, Christopher Spurney ${ }^{\mathrm{e}}$, Justin Godown ${ }^{\mathrm{a}}$, Meng Xu ${ }^{f}$, \\ James C Slaughter ${ }^{f}$, and Larry W Markham ${ }^{\mathrm{a}, \mathrm{g}}$ \\ aThomas P Graham Division of Pediatric Cardiology, Department of Pediatrics, Vanderbilt \\ University Medical Center, Nashville, TN 37232 USA. \\ ${ }^{b}$ Children's Hospital Association, Lenexa, KS 66219 USA. \\ 'Division of Pediatric Neurology, Department of Pediatrics, Vanderbilt University Medical Center, \\ Nashville, TN 37232 USA. \\ 'Division of Pediatric Cardiology, Nationwide Children's Hospital and The Ohio State University, \\ Columbus, $\mathrm{OH} 43205$ USA. \\ eDivision of Cardiology, Children's National Heart Institute, Children's National Health System, \\ Washington, D.C. 20010, USA. \\ fDepartment of Biostatistics, Vanderbilt University Medical Center, Nashville, TN 37232 USA. \\ gDivision of Cardiology, Department of Pediatrics, Riley Hospital for Children at Indiana University \\ Health, Indianapolis, IN 46202 USA.
}

\begin{abstract}
Background-Outcome analyses in large administrative databases are ideal for rare diseases such as Becker and Duchenne muscular dystrophy. Unfortunately, Becker and Duchenne do not yet have specific ICD-9/ICD-10 codes. We hypothesized that an algorithm could accurately identify these patients within administrative data and improve assessment of cardiovascular morbidity.
\end{abstract}

\footnotetext{
Corresponding Author: Jonathan H. Soslow, M.D., M.S.C.I., Assistant Professor, Pediatrics, Thomas P. Graham, Jr. Division of Pediatric Cardiology, Monroe Carell Jr. Children's Hospital at Vanderbilt, 2200 Children's Way, Suite 5230, Doctors' Office Tower, Nashville, TN 37232-9119, Phone: (615) 322-7447, Fax: (615) 322-2210, jonathan.h.soslow@ vanderbilt.edu,.

Matt.Hall@childrenshospitals.org, william.b.burnette@Vanderbilt.Edu, Kan.Hor@nationwidechildrens.org,

Joanne.Chisolm@ @ationwidechildrens.org, CSpurney@ childrensnational.org, justin.godown@ vanderbilt.edu, meng.xu@ vumc.org, james.c.slaughter@Vanderbilt.Edu, lwmarkha@iu.edu.

CONFLICTS OF INTEREST

None

ETHICAL STANDARDS

The authors assert that all procedures contributing to this work comply with the ethical standards of the relevant national guidelines on human experimentation Belmont Report and with the Helsinki Declaration of 1975, as revised in 2008, and has been approved by the institutional committees the Institutional Review Boards of Vanderbilt University Medical Center, Nationwide Children's Hospital,and Children's National Medical Center approved this study.
} 
Methods-Hospital discharges ( $\mathrm{n}=13,189)$ for patients with muscular dystrophy (ICD-9 359.1) were identified in the Pediatric Health Information System database. An identification algorithm was created then validated at 3 institutions. Multivariable generalized linear mixed effects models used to estimate the associations of length of stay, hospitalization cost, and 14-day readmission with age, encounter severity, and respiratory disease accounting for clustering within a hospital.

Results-The identification algorithm improved identification of Becker and Duchenne patients from 55\% (359.1 alone) to 77\%. On bivariate analysis, left ventricular dysfunction and arrhythmia were associated with increased cost of hospitalization, length of stay, and mortality ( $p<0.001)$. After adjustment, Becker and Duchenne patients with left ventricular dysfunction and arrhythmia had increased length of stay (rate ratio 1.4 and 1.2, $\mathrm{p}<0.001$ and $\mathrm{p}=0.004$ ) and cost (rate ratio 1.4 and 1.4 , both $\mathrm{p}<0.001$ ).

Conclusions-Our algorithm accurately identifies patients with Becker and Duchenne and can be used for future analysis of administrative data. Our analysis demonstrates the significant effects of cardiovascular disease on length of stay and hospitalization cost in Becker and Duchenne patients. Better recognition of the contribution of cardiovascular disease during hospitalization with earlier, more intensive evaluation and therapy may help improve outcomes in this patient population.

\section{Keywords}

Duchenne muscular dystrophy; Becker muscular dystrophy; Pediatric Health Information System; Left ventricular dysfunction; Arrhythmia; Cardiomyopathy

\section{INTRODUCTION}

Becker and Duchenne muscular dystrophy are the most common muscular dystrophies, with an incidence of 1 in 18500 and 1 in 4700 live male births, respectively. ${ }^{1,2}$ Patients with Becker and Duchenne muscular dystrophy have a mutation in the DMD gene that leads to a deficiency or absence of dystrophin protein. ${ }^{3,4}$ This results in skeletal muscle weakness and loss of ambulation, usually in the second decade of life in Duchenne muscular dystrophy and later in life in the milder Becker muscular dystrophy. Both reduced and absent dystrophin also leads to the development of cardiomyopathy resulting in progressive left ventricular dysfunction. 5,6

Understanding of the clinical importance of Becker and Duchenne cardiomyopathy has increased significantly over the past few decades. This is primarily due to improvements in care, which have increased life expectancy in patients with Duchenne muscular dystrophy and unmasked the cardiovascular phenotype. ${ }^{7}$ The preponderance of Becker and Duchenne research focuses on skeletal muscle weakness, but cardiovascular disease is now one of the leading causes of death in patients with Duchenne muscular dystrophy. ${ }^{8}$ Highlighting the effects of cardiovascular disease on inpatient morbidity (length of stay, cost of hospitalization, readmission rate) in patients with Becker and Duchenne may help improve recognition of the problems associated with cardiovascular disease. Moreover, identifying risk factors leading to increased length of stay, cost of hospitalization, and readmission rates 
in this population can increase recognition and potentially improve outcomes in future admissions.

Because Becker and Duchenne muscular dystrophy are relatively rare, the true impact of cardiovascular disease can be difficult to determine. Research using large administrative databases can overcome these issues. However, using the International Classification of Disease (ICD)-9 billing codes, Becker and Duchenne muscular dystrophy are coded as 359.1, a non-specific code that also encompasses multiple other neuromuscular diagnoses (Table 1). This non-specific coding can make analysis problematic. Indeed, a recent manuscript using the Pediatric Health Information System database to evaluate the effect of cardiovascular disease in Duchenne muscular dystrophy was limited by this non-specific coding. ${ }^{9}$ We hypothesized that, by creating a novel list of exclusion criteria which could be validated, patients with Becker and Duchenne muscular dystrophy could be more accurately identified within an administrative and billing database, allowing for the current study and future assessments in this patient population. To demonstrate the potential utility of this technique, we used the Pediatric Health Information System database to assess cardiovascular risk factors associated with increased length of stay, cost of hospitalization, and 14-day readmission in patients with Becker and Duchenne muscular dystrophy.

\section{MATERIALS AND METHODS}

\subsection{Pediatric Health Information System Database and Identification Algorithm}

This multicenter retrospective cohort study utilized administrative data from the Pediatric Health Information System database. The database contains inpatient, emergency department, ambulatory surgery, and observation encounters from 49 tertiary children's hospitals in the United States. Data are de-identified at the time of submission and are subjected to reliability and validity checks by participating hospitals, Children's Hospital Association (Lenexa, KS), and Truven Health Analytics (Ann Arbor, MI) before inclusion in the database. Patients are assigned up to 41 ICD-9/10 diagnosis and procedure codes, and consistently encrypted medical record numbers allow longitudinal tracking of patients across encounters to the same hospital. The Pediatric Health Information System database represents approximately $15 \%$ of the national pediatric hospitalizations and $46.4 \%$ of children's hospitals' total volume.

The Institutional Review Boards of Vanderbilt University Medical Center, Nationwide Children's Hospital, and Children's National Medical Center approved this study. Using ICD-9 diagnosis codes (conversion to ICD-10 codes occurred in the last quarter of 2015), an algorithm of exclusion criteria termed the "identification algorithm" was created to improve the identification of patients with Becker and Duchenne muscular dystrophy. The effectiveness of the algorithm was evaluated by "re-identification" of patients from three sites. The electronic medical record was queried to determine the diagnosis and gender of all patients with an ICD-9 code of 359.1 and the diagnosis of the remaining patients after the identification algorithm was applied.

The algorithm was constructed to optimize the specificity of identifying patients with Becker and Duchenne muscular dystrophy. Given the size of the Pediatric Health Information 
System database, investigators determined that incorrectly excluding patients with Becker and Duchenne muscular dystrophy was preferable to incorrectly including patients with an alternate diagnosis. The goal at the outset of the study was to construct an algorithm where at least $80 \%$ of remaining patients carried a diagnosis of Becker and Duchenne muscular dystrophy. All patients with an ICD-9 of 359.1 were initially included. In order to eliminate errors in coding, patients with a change in primary diagnosis to a different 359.x code were excluded. A set of clinical characteristics was created to exclude neuromuscular conditions other than Becker and Duchenne muscular dystrophy. Briefly, patients with severe disease at a young age felt unlikely to be due to Becker or Duchenne were eliminated (early mortality, ventilatory support, or cardiovascular disease). Because the majority of Becker and Duchenne patients do not require gastrostomy tubes until later in life, and only $15 \%$ of patients were older than 18 in this cohort, all patients with gastrostomy tubes were removed. Other neurologic diagnoses that could have been mistakenly coded as 359.1 were removed, as were diagnoses that came up in an initial search of 359.1 at one institution. Patients undergoing heart transplantation were not excluded as transplantation has been performed in the Becker muscular dystrophy population.

\subsection{Primary Analysis}

The primary analysis was performed on the entire cohort ( 49 hospitals) after application of the identification algorithm. Discharges in patients with muscular dystrophy were identified in Pediatric Health Information System from January 1, 2003 - September 30, 2015 (chosen based on transition from ICD-9 to ICD-10). Patients with left ventricular dysfunction and arrhythmia were identified using ICD-9 codes (Supplementary Table S1). Patients requiring respiratory support (Table S1) were also identified. Discharges that occurred under 1 year of age were eliminated from the analysis as these admissions were more likely to reflect neonatal and infant hospitalizations unrelated to the diagnosis of Becker and Duchenne muscular dystrophy. The primary outcome measures included length of stay, cost of hospitalization (estimated from charges using hospital/year specific ratios of cost to charge), 14-day all-cause readmission, and in-hospital mortality.

\subsection{Statistical Analysis}

To assess the effectiveness of the identification algorithm, the percentage of the patients with Becker and Duchenne muscular dystrophy before and after application of the algorithm was calculated for the 3 sites. The percentage of patients with Becker and Duchenne muscular dystrophy who were incorrectly excluded was also calculated.

For the primary analysis on the entire Pediatric Health Information System cohort, pertinent demographic information was summarized and bivariate analyses were performed to assess the relationships of each demographic and length of stay (stratified by $<2$ days vs. $2+$ days) using chi-square and Wilcoxon Rank Sum tests. Next, separate generalized linear mixed effects models assuming either an exponential distribution (length of stay and cost) or a binomial distribution (14-day readmission and mortality) for the outcome were used to estimate the adjusted associations with age, encounter severity, and respiratory disease. A random intercept was used to account for correlation arising from taking repeated measurements on the same hospital. Results are summarized using the rate ratio or odds 
ratio with corresponding 95\% confidence intervals. Statistical analyses were performed using SAS v.9.4 (SAS Institute, Cary, NC), and a p-value < 0.05 was considered statistically significant.

\section{RESULTS}

\subsection{Identification Algorithm}

The final identification algorithm is listed in Table 2. At the 3 Pediatric Health Information System sites, we performed a detailed assessment of our algorithm by re-identifying the 289 patients with an ICD-9 of 359.1 and determining true diagnosis using the local electronic medical record. Of the 289 patients, 55\% (158) had a diagnosis of Becker and Duchenne muscular dystrophy prior to the application of the identification algorithm. Excluding female patients increased this to $68 \%$ (158 of 233). Applying the identification algorithm increased the accuracy of correct Becker and Duchenne muscular dystrophy diagnosis to 77\% (131 of 170) by excluding 27 patients with Becker and Duchenne muscular dystrophy (17\%) and 91 patients without Becker and Duchenne muscular dystrophy (70\% of non-Becker and nonDuchenne muscular dystrophy patients). Of note, two sites provided a breakdown of Becker muscular dystrophy vs Duchenne muscular dystrophy, and only $5 \%$ of included patients at those sites had Becker muscular dystrophy. We hypothesize that this preponderance of Duchenne muscular dystrophy is due to the relatively higher frequency in the general population and earlier cardiomyopathy as well as a lower frequency of admission in children with Becker muscular dystrophy.

\subsection{Demographics}

In the entire Pediatric Health Information System cohort, a total of 3,430 unique patients with 13,189 discharges from 49 hospitals were identified with an ICD-9 of 359.1. After application of the identification algorithm, 1,916 patients and 4014 discharges remained.

The majority of discharges (89.5\%) were to home, $4 \%$ were discharged to a skilled facility, $3.1 \%$ to home health service, and $2.2 \%$ (90 discharges) died in the hospital (4.7\% of patients). As expected, neuromuscular and cardiovascular codes were the most common, with $90 \%$ of discharges having at least one neuromuscular code and 30\% having at least one cardiovascular code. In addition, $22 \%$ had a code for congenital or genetic defects, $21 \%$ had a code suggesting technology dependence, and $7 \%$ had a code denoting respiratory disease. A total of $26 \%$ of discharges had a diagnosis of left ventricular dysfunction, $8 \%$ arrhythmia, and $21 \%$ respiratory disease. Further demographic data are reported in Table 3.

\subsection{Primary Analysis}

Bivariate analysis for the predictors left ventricular dysfunction, arrhythmia, and respiratory disease for the outcomes of length of stay, cost of hospitalization, 14-day readmission, and mortality are shown in Table 4. We also considered adjusted models controlling for age, encounter severity, respiratory disease, and hospital. The adjusted association of predictors with mortality was not estimated because there were too few events to support a multivariable model. However, for length of stay we found significant adjusted association with left ventricular dysfunction (rate ratio $=1.4,95 \%$ CI $[1.3,1.5], \mathrm{p}<0.001$ ), arrhythmia 
(rate ratio $=1.2,95 \% \mathrm{CI}[1.1,1.4], \mathrm{p}=0.004)$, and respiratory disease (rate ratio $=1.6,95 \%$ CI $[1.5,1.8], \mathrm{p}<0.001$ ) (Table 5). We also found significant adjusted association for cost of hospitalization with left ventricular dysfunction (rate ratio $=1.2,95 \%$ CI $[1.1,1.3]$, $\mathrm{p}<0.001$ ), arrhythmia (rate ratio $=1.4,95 \%$ CI $[1.2,1.6], \mathrm{p}<0.001$ ), and respiratory disease (rate ratio $=1.4,95 \%$ CI $[1.3,1.5], \mathrm{p}<0.001)$. Becker and Duchenne muscular dystrophy patients with respiratory disease had decreased rates of 14-day readmission (odds ratio $=0.6$, 95\% CI [0.3,1], $\mathrm{p}=0.045$ ), while left ventricular dysfunction (odds ratio $=1,95 \% \mathrm{CI}$ $[0.7,1.3], \mathrm{p}=0.780$ ) and arrhythmia (odds ratio $=1.5,95 \%$ CI $[0.9,2.5], \mathrm{p}=0.128$ ) were not significantly associated with readmission. The combination of arrhythmia and left ventricular dysfunction (cardiovascular disease) demonstrated similar effects for hospitalization cost and length of stay (Supplementary Table S2).

\section{DISCUSSION}

These results demonstrate that: 1) the application of our identification algorithm confirmed by direct validation significantly increases the specificity for Becker and Duchenne muscular dystrophy in the Pediatric Health Information System database and 2) left ventricular dysfunction, arrhythmia, and respiratory disease play significant, independent roles in predicting inpatient morbidity and, in the bivariate model, mortality. Given the rarity of Becker and Duchenne muscular dystrophy, a method that allows researchers to leverage large databases is critical to improving outcomes. Analysis of the Pediatric Health Information System database may help identify areas of improvement that could lead to a decrease in the costs of hospitalization for patients with Becker and Duchenne muscular dystrophy. Moreover, these data can serve as a baseline appraisal, allowing for the assessment of future shifts in outcome resulting from modifications in therapies or changes to standards of care in Becker and Duchenne muscular dystrophy. To allow for future analyses, we have translated our exclusion criteria to ICD-10 diagnostic codes (Supplementary Table S3).

The ICD-9 billing code that includes Becker and Duchenne muscular dystrophy also includes many other diagnoses. Fortunately, this will be rectified in October of 2018 with a new ICD-10 code. However, given that this manuscript used over 12 years of data from the Pediatric Health Information System, it will take an extended period of time for that change to be relevant. The algorithm reported here will remain critical for future analyses and will facilitate analysis of historical trends. In addition, similar methods can be used to evaluate morbidity and mortality due to other forms of muscular dystrophy coded as 359.1.

These data further emphasize the importance of cardiovascular disease in Becker and Duchenne muscular dystrophy. It is notable that $30 \%$ of the patients in this analysis had at least one cardiovascular code while only $7 \%$ had a code for respiratory disease. Cardiovascular disease has become increasingly recognized as a major contributor of mortality. ${ }^{8,10}$ Punnoose et al recently evaluated the association between cardiovascular disease and morbidity and mortality in the Pediatric Health Information System database, though their analysis was limited by the poor specificity of ICD-9 coding for Duchenne muscular dystrophy. ${ }^{9}$ Their study also demonstrated that ventricular tachycardia and heart failure, as well as chronic ventilator use, were risk factors for the combined outcome of 
cardiac arrest or death. Their exclusions likely improved specificity for Duchenne muscular dystrophy but are less extensive than the algorithm we present here, and they were unable to directly validate the effectiveness of their exclusion criteria. The prevalence of cardiomyopathy increases significantly as Becker and Duchenne muscular dystrophy patients age, with previous studies suggesting that $>60 \%$ of patients over 18 years of age have cardiomyopathy. ${ }^{5,11}$ Unfortunately, while official recommendations state that echocardiographic screening should begin by 6 years of age, Spurney et al demonstrated that a surprisingly large portion of Duchenne muscular dystrophy patients had not undergone echocardiography by 10 years of age. ${ }^{11}$ Moreover,that study revealed that over half of Duchenne muscular dystrophy patients with a diagnosis of cardiomyopathy were not prescribed appropriate anti-congestive therapy. Taken together, these data suggest that the focus of care in Becker and Duchenne muscular dystrophy must shift towards earlier detection and prevention of cardiovascular disease. Raising awareness of the risks of cardiovascular disease in Becker and Duchenne muscular dystrophy patients admitted to the hospital may lead to earlier recognition and more aggressive therapy, eventually improving outcomes.

Our data confirm that respiratory disease continues to play a significant role in inpatient morbidity and mortality in patients with Becker and Duchenne muscular dystrophy. Multiple authors have reported the improved life expectancy associated with ventilation in Duchenne muscular dystrophy. ${ }^{7,8,12}$ While survival has increased significantly with these interventions, respiratory disease of any kind, including acute respiratory complications and the need for chronic ventilation, significantly increased the odds of a longer length of stay and higher cost of hospitalization. Moreover, the complex interactions between cardiovascular and respiratory disease likely plays an important role in Becker and Duchenne muscular dystrophy inpatient morbidity.

Multiple reports have demonstrated the increased cost of both Duchenne and Becker muscular dystrophy compared with the general population. Thayer et al demonstrated a 10fold increase in costs in a small cohort of 75 Duchenne muscular dystrophy patients by analyzing claims from a single health plan. ${ }^{13}$ Surveys of families in multiple countries have demonstrated increases in both healthcare costs and out of pocket costs to families of Becker and Duchenne muscular dystrophy patients. ${ }^{14-16}$ In general, costs increase with increasing age/progression of disease. ${ }^{14,15}$ Costs have also been shown to be increased in Duchenne muscular dystrophy patients with tracheostomy compared to those on continuous noninvasive ventilation. ${ }^{17}$ However, only our data and that of Punnoose have stratified costs based on cardiovascular disease. ${ }^{9}$ Awareness of the increased cost associated with both arrhythmia and left ventricular dysfunction could allow for improvement in Becker and Duchenne muscular dystrophy costs with earlier and more aggressive detection and therapy. Not surprisingly, our data also demonstrated significantly increased length of stay and cost of hospitalization with advancing age in Becker and Duchenne muscular dystrophy, even when correcting for arrhythmia, left ventricular dysfunction, and respiratory disease. 


\title{
4.1 Limitations
}

This study does have inherent limitations. As with any large database, data could be incomplete, incorrect, or missing. The Pediatric Health Information System does not capture events that occur outside a hospital encounter and does not include physician fees as part of the cost analysis. In addition, the Pediatric Health Information System only captures events that occur in children's hospitals. Specific to this study, our algorithm prioritized identification of Becker and Duchenne muscular dystrophy patients with the knowledge that some patients would be incorrectly excluded. However, re-identification demonstrated that $17 \%$ of Becker and Duchenne muscular dystrophy patients were incorrectly excluded while $70 \%$ of non-Becker and Duchenne muscular dystrophy were excluded. The algorithm we present to better identify Becker and Duchenne muscular dystrophy patients does still include patients without Becker and Duchenne muscular dystrophy, but this number is significantly lower after our exclusion criteria and we feel the advantages of using larger numbers for this and future studies will outweigh this limitation. We also evaluated an algorithm that increased the identification of Becker and Duchenne muscular dystrophy patients to 79\% (Supplementary Table 4), but this algorithm excluded a much larger number of patients with Becker's and Duchenne's (35\%), so the less aggressive algorithm was used for the final analysis. Of note, the results of the primary analysis were not significantly different between algorithms.

\subsection{Conclusions}

We present an algorithm for improving identification of Becker and Duchenne muscular dystrophy patients using an administrative database. Initial analysis demonstrated a large number of Becker and Duchenne muscular dystrophy patients with cardiovascular codes and increased cost and length of stay in patients with both arrhythmia and left ventricular dysfunction. This algorithm can be used to analyze other outcomes of interest in patients with Becker and Duchenne muscular dystrophy and can also serve as a baseline to assess changes in therapy over time.

\section{Supplementary Material}

Refer to Web version on PubMed Central for supplementary material.

\section{ACKNOWLEDGEMENTS}

\author{
FINANCIAL SUPPORT
}

Research reported in this publication was supported by the National Heart, Lung, and Blood Institute of the National Institutes of Health under Award Number K23HL123938 (Bethesda, MD) (Soslow). The content is solely the responsibility of the authors and does not necessarily represent the official views of the National Institutes of Health.

This project was supported by the Fighting Duchenne Foundation and the Fight DMD/Jonah \& Emory Discovery Grant (Nashville, TN) (Markham).

The sponsors and funders had no role in the design and conduct of the study or in the collection, analysis, and interpretation of the data, or in the preparation, review, or approval of the manuscript. 


\section{REFERENCES}

1. Dooley J, Gordon KE, Dodds L and MacSween J. Duchenne muscular dystrophy: a 30-year population-based incidence study. Clin Pediatr (Phila) 2010; 49: 177-179. [PubMed: 20080524]

2. Bushby KM, Thambyayah M and Gardner-Medwin D. Prevalence and incidence of Becker muscular dystrophy. Lancet 1991; 337: 1022-1024. [PubMed: 1673177]

3. Moser H Duchenne muscular dystrophy: pathogenetic aspects and genetic prevention. Hum Genet 1984; 66: 17-40. [PubMed: 6365739]

4. Hoffman EP, Brown RH, Jr. and Kunkel LM. Dystrophin: the protein product of the Duchenne muscular dystrophy locus. Cell 1987; 51: 919-928. [PubMed: 3319190]

5. Nigro G, Comi LI, Politano L and Bain RJ. The incidence and evolution of cardiomyopathy in Duchenne muscular dystrophy. International journal of cardiology 1990; 26: 271-277. [PubMed: 2312196]

6. American Academy of Pediatrics Section on C and Cardiac S. Cardiovascular health supervision for individuals affected by Duchenne or Becker muscular dystrophy. Pediatrics 2005; 116: 1569-1573. [PubMed: 16322188]

7. Eagle M, Baudouin SV, Chandler C, Giddings DR, Bullock R and Bushby K. Survival in Duchenne muscular dystrophy: improvements in life expectancy since 1967 and the impact of home nocturnal ventilation. Neuromuscul Disord 2002; 12: 926-929. [PubMed: 12467747]

8. Bach JR and Martinez D. Duchenne muscular dystrophy: continuous noninvasive ventilatory support prolongs survival. Respir Care 2011; 56: 744-750. [PubMed: 21333078]

9. Punnoose AR, Kaltman JR, Pastor W, McCarter R, He J and Spurney CF. Cardiac Disease Burden and Risk of Mortality in Hospitalized Muscular Dystrophy Patients. Pediatr Cardiol 2016.

10. Connuck DM, Sleeper LA, Colan SD et al. Characteristics and outcomes of cardiomyopathy in children with Duchenne or Becker muscular dystrophy: a comparative study from the Pediatric Cardiomyopathy Registry. Am Heart J 2008; 155: 998-1005. [PubMed: 18513510]

11. Spurney C, Shimizu R, Morgenroth LP et al. Cooperative International Neuromuscular Research Group Duchenne Natural History Study demonstrates insufficient diagnosis and treatment of cardiomyopathy in Duchenne muscular dystrophy. Muscle Nerve 2014; 50: 250-256. [PubMed: 24395289]

12. Eagle M, Bourke J, Bullock R et al. Managing Duchenne muscular dystrophy--the additive effect of spinal surgery and home nocturnal ventilation in improving survival. Neuromuscul Disord 2007; 17: 470-475. [PubMed: 17490881]

13. Thayer S, Bell C and McDonald CM. The Direct Cost of Managing a Rare Disease: Assessing Medical and Pharmacy Costs Associated with Duchenne Muscular Dystrophy in the United States. J Manag Care Spec Pharm 2017; 23:633-641. [PubMed: 28530521]

14. Teoh LJ, Geelhoed EA, Bayley K, Leonard H and Laing NG. Health care utilization and costs for children and adults with duchenne muscular dystrophy. Muscle Nerve 2016; 53: 877-884. [PubMed: 26562484]

15. Schreiber-Katz O, Klug C, Thiele S et al. Comparative cost of illness analysis and assessment of health care burden of Duchenne and Becker muscular dystrophies in Germany. Orphanet J Rare Dis 2014; 9: 210. [PubMed: 25519771]

16. Landfeldt E, Lindgren P, Bell CF et al. The burden of Duchenne muscular dystrophy: an international, cross-sectional study. Neurology 2014; 83: 529-536. [PubMed: 24991029]

17. Bach JR, Tran J and Durante S. Cost and physician effort analysis of invasive vs. noninvasive respiratory management of Duchenne muscular dystrophy. Am J Phys Med Rehabil 2015; 94: 474-482. [PubMed: 25741618] 
Table 1:

List of Diagnoses Included in ICD-9 Code 359.1

\begin{tabular}{ll}
\hline Facioscapulohumeral muscular dystrophy & Hereditary, progressive muscular dystrophy \\
Landouzy-Déjérine & Leyden-Möbius \\
Pseudohypertrophic muscular dystrophy & Distal muscular dystrophy \\
Duchenne's muscular dystrophy & Fascioscapulohumeral muscular dystrophy \\
Duchenne-Griesinger & Limb-girdle muscular dystrophy \\
Erb (-Landouzy) & Ocular muscular dystrophy \\
Hereditary neuromuscular NEC & Oculopharyngeal muscular dystrophy \\
Duchenne's pseudohypertrophy, muscles & Pelvicrural atrophic muscular dystrophy \\
Erb's dystrophy & Progressive ophthalmoplegic muscular dystrophy \\
Gower's muscular dystrophy & Scapuloperoneal ophthalmoplegic muscular dystrophy \\
Emery Dreifuss muscular dystrophy & Becker's muscular dystrophy \\
Kearns-Sayre syndrome (disorder) & Mitochondrial ocular myopathy \\
Muscular dystrophy & Neuropathic muscular dystrophy \\
Restrictive lung disease due to muscular dystrophy & Congenital myopathy \\
Congenital muscular dystrophy & \\
\hline
\end{tabular}


Table 2:

\section{Identification Algorithm}

\begin{tabular}{|c|c|c|}
\hline & $\begin{array}{c}\text { Patients } \\
\text { Excluded }\end{array}$ & $\begin{array}{c}\text { Patients } \\
\text { Remaining }\end{array}$ \\
\hline \multicolumn{3}{|l|}{ Inclusion } \\
\hline ICD-9=359.1 & & 3430 \\
\hline \multicolumn{3}{|l|}{ Exclusions } \\
\hline $\begin{array}{l}\text { Cerebral degenerations (330.x), Spinocerebellar disease (334.x), Anterior horn cell disease (335.x), or Hereditary } \\
\text { and idiopathic peripheral neuropathy (356.x) }\end{array}$ & 387 & 3043 \\
\hline Changed to another $359 . \mathrm{x}$ code & 405 & 2638 \\
\hline G-tube ${ }^{a}$ & 458 & 2180 \\
\hline Ophthalmoplegia & 2 & 2178 \\
\hline Pigmentary retinopathy & 0 & 2178 \\
\hline Mortality age $<5$ & 8 & 2170 \\
\hline Ventilation $<5$ years of age (tracheostomy or CPAP/BIPAP ${ }^{b}$ ) & 0 & 2170 \\
\hline $\operatorname{AICD}^{c}<10$ years of age & 1 & 2169 \\
\hline Arrhythmia $<5$ years of age & 7 & 2162 \\
\hline Cardiovascular disease $<5$ years of age & 6 & 2156 \\
\hline Female & 224 & 1932 \\
\hline Age $<1$ year at time of only encounter & 16 & 1916 \\
\hline \multicolumn{3}{|l|}{ gastrostomy tube (G-tube) } \\
\hline
\end{tabular}


Table 3:

\section{Demographics}

\begin{tabular}{|c|c|c|c|}
\hline & & Prior to Exclusions & Post Exclusions \\
\hline N Discharges & & 13189 & 4014 \\
\hline N Patients & & 3430 & 1916 \\
\hline Discharge contained & & $7538(57.2 \%)$ & $3522(87.7 \%)$ \\
\hline \multicolumn{4}{|l|}{ ICD-9 code 359.1} \\
\hline Age: Median [IQR] & & $13[6,17]$ & $14[11,17]$ \\
\hline \multirow[t]{6}{*}{ Age } & $<1$ & $640(4.9 \%)$ & \\
\hline & $1-4$ & $1966(14.9 \%)$ & $159(4 \%)$ \\
\hline & $5-9$ & $2105(16 \%)$ & $504(12.6 \%)$ \\
\hline & $10-14$ & $3344(25.4 \%)$ & $1431(35.7 \%)$ \\
\hline & $15-18$ & $3124(23.7 \%)$ & $1293(32.2 \%)$ \\
\hline & $19+$ & $2010(15.2 \%)$ & $627(15.6 \%)$ \\
\hline Gender & Male & $9746(73.9 \%)$ & $4014(100 \%)$ \\
\hline \multirow[t]{6}{*}{ Race } & Non-Hispanic & $7900(59.9 \%)$ & $2590(64.5 \%)$ \\
\hline & White & & \\
\hline & Non-Hispanic Black & $1139(8.6 \%)$ & $289(7.2 \%)$ \\
\hline & Hispanic & $2826(21.4 \%)$ & $730(18.2 \%)$ \\
\hline & Asian & $313(2.4 \%)$ & $81(2 \%)$ \\
\hline & Other & $1011(7.7 \%)$ & $324(8.1 \%)$ \\
\hline \multirow[t]{6}{*}{ Discharge Disposition } & Home Health & $692(5.2 \%)$ & $123(3.1 \%)$ \\
\hline & Service & & \\
\hline & Home & $11690(88.6 \%)$ & $3593(89.5 \%)$ \\
\hline & Skilled Facility & $385(2.9 \%)$ & $159(4 \%)$ \\
\hline & Died & $210(1.6 \%)$ & $90(2.2 \%)$ \\
\hline & Other & $212(1.6 \%)$ & $49(1.2 \%)$ \\
\hline \multirow[t]{14}{*}{ CCCs v.2.0 } & Neuromuscular & $11202(84.9 \%)$ & $3594(89.5 \%)$ \\
\hline & Cardiovascular & $3189(24.2 \%)$ & $1192(29.7 \%)$ \\
\hline & Disease & & \\
\hline & Respiratory & $3248(24.6 \%)$ & $272(6.8 \%)$ \\
\hline & Renal & $458(3.5 \%)$ & $65(1.6 \%)$ \\
\hline & Gastrointestinal & $4484(34 \%)$ & $74(1.8 \%)$ \\
\hline & Hematology and & $224(1.7 \%)$ & $61(1.5 \%)$ \\
\hline & Immunodeficiency & & \\
\hline & Metabolic & $1125(8.5 \%)$ & $224(5.6 \%)$ \\
\hline & $\begin{array}{l}\text { Congenital or } \\
\text { genetic defect }\end{array}$ & $3642(27.6 \%)$ & $896(22.3 \%)$ \\
\hline & Malignancy & $158(1.2 \%)$ & $68(1.7 \%)$ \\
\hline & Technology & $6432(48.8 \%)$ & $849(21.2 \%)$ \\
\hline & Dependence & & \\
\hline & Transplant & $213(1.6 \%)$ & $106(2.6 \%)$ \\
\hline
\end{tabular}




\begin{tabular}{llll}
\hline & & Prior to Exclusions & Post Exclusions \\
\hline & Any & $12435(94.3 \%)$ & $3734(93 \%)$ \\
Length of Stay: & & $3[1,7]$ & $2[1,6]$ \\
Median [IQR] & & & \\
Length of Stay & a. $0-1$ days & $4007(30.4 \%)$ & $1579(39.3 \%)$ \\
& b. $2-3$ days & $3071(23.3 \%)$ & $811(20.2 \%)$ \\
& c. $4-6$ days & $2367(17.9 \%)$ & $697(17.4 \%)$ \\
& d. 27 days & $3744(28.4 \%)$ & $927(23.1 \%)$ \\
Decreased left ventricular function & & $2419(18.3 \%)$ & $1050(26.2 \%)$ \\
Arrhythmia & & $938(7.1 \%)$ & $301(7.5 \%)$ \\
Respiratory support & & $4944(37.5 \%)$ & $853(21.3 \%)$ \\
\hline
\end{tabular}



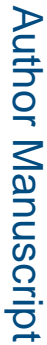

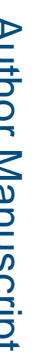

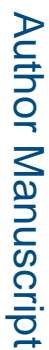

Cardiol Young. Author manuscript; available in PMC 2019 September 01. 
Table 4A:

Bivariate Analysis for Decreased Left Ventricular (LV) Function

\begin{tabular}{lccc}
\hline \multicolumn{3}{c}{ Decreased LV Function } \\
\hline No & Yes & p \\
\hline N Discharges & $2964(73.8 \%)$ & $1050(26.2 \%)$ & \\
Length of Stay & 2 & 5 & $<.001$ \\
(days) & {$[1,5]^{a}$} & {$[2,10]$} & \\
Cost (dollars) & 6119 & 16162 & $<.001$ \\
& {$[3317,17275]$} & {$[7038,45282]$} & \\
14 Day & & & 0.557 \\
Readmission & $158(5.3 \%)$ & $61(5.8 \%)$ & \\
Mortality & $29(1 \%)$ & $61(5.8 \%)$ & $<0.001$ \\
\hline
\end{tabular}

Interquartile range 
Table 4B:

Bivariate Analysis for Arrhythmia

\begin{tabular}{lccc}
\hline \multicolumn{3}{c}{ Arrhythmia } \\
\hline No & Yes & p \\
\hline N Discharges & $3713(92.5 \%)$ & $301(7.5 \%)$ & \\
Length of Stay & 2 & 5 & $<.001$ \\
(days) & {$[1,6]$} & {$[2,13]$} & \\
Cost (dollars) & 7324 & 25189 & $<.001$ \\
& {$[3585,20906]$} & {$[9330,61637]$} & \\
14 Day & & & 0.046 \\
Readmission & $195(5.3 \%)$ & $24(8 \%)$ & \\
Mortality & $45(1.2 \%)$ & $45(15 \%)$ & $<0.001$ \\
\hline
\end{tabular}

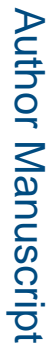

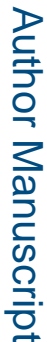

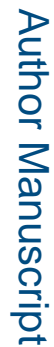

Cardiol Young. Author manuscript; available in PMC 2019 September 01. 
Table 4C:

Bivariate analysis for respiratory disease

\begin{tabular}{lccc}
\hline \multicolumn{3}{c}{ Respiratory Disease } \\
\hline No & Yes & p \\
\hline N Discharges & $3161(78.7 \%)$ & $853(21.3 \%)$ & \\
Length of Stay & 2 & 6[ & $<.001$ \\
(days) & {$[1,5]$} & $3,13]$ & \\
Cost (dollars) & 6161 & 22222 & $<.001$ \\
& {$[3390,15860]$} & {$[9024,61141]$} & \\
14 Day & & & 0.347 \\
Readmission & $178(5.6 \%)$ & $41(4.8 \%)$ & \\
Mortality & $30(0.9 \%)$ & $60(7 \%)$ & $<0.001$ \\
\hline
\end{tabular}



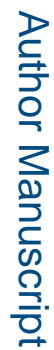

Cardiol Young. Author manuscript; available in PMC 2019 September 01. 
Table 4D:

Number of Discharges with Each Combination of Exposure

\begin{tabular}{cccccc}
\hline Decreased left ventricular function/cardiomyopathy & \multicolumn{4}{c}{ Respiratory } \\
\hline & Arrhythmia & support & N & $\%$ \\
\hline No & No & No & 2440 & 60.8 \\
\hline No & No & Yes & 416 & 10.3 \\
\hline No & Yes & No & 72 & 1.8 \\
\hline No & Yes & Yes & 36 & 0.9 \\
\hline Yes & No & No & 541 & 13.5 \\
\hline Yes & No & Yes & 316 & 7.9 \\
\hline Yes & Yes & No & 108 & 2.7 \\
\hline Yes & Yes & Yes & 85 & 2.1 \\
\hline
\end{tabular}


Table 5A:

Generalized linear mixed effects model with LOS as outcome measure

\begin{tabular}{|c|c|c|c|}
\hline & & LOS & \\
\hline & & Rate Ratio (95\% CI) & $\mathbf{p}$ \\
\hline \multirow[t]{5}{*}{ Age } & $1-4$ & Reference & \\
\hline & $5-9$ & $1(0.8,1.1)$ & 0.629 \\
\hline & $10-14$ & $1.2(1,1.4)$ & 0.034 \\
\hline & $15-18$ & $1.4(1.1,1.6)$ & 0.001 \\
\hline & $19+$ & $1.4(1.2,1.7)$ & $<.001$ \\
\hline \multirow[t]{2}{*}{ Decreased LV function } & Yes & $1.4(1.3,1.5)$ & $<.001$ \\
\hline & No & Reference & \\
\hline \multirow[t]{2}{*}{ Arrhythmia } & Yes & $1.2(1.1,1.4)$ & 0.004 \\
\hline & No & Reference & \\
\hline \multirow[t]{2}{*}{ Respiratory support } & Yes & $1.6(1.5,1.8)$ & $<.001$ \\
\hline & No & Reference & \\
\hline
\end{tabular}

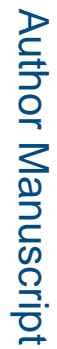

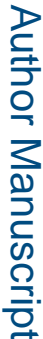

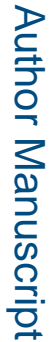

로을

Cardiol Young. Author manuscript; available in PMC 2019 September 01. 
Table 5B:

Generalized linear mixed effects model with cost of hospitalization as outcome measure




Table 5C:

Generalized estimating equation with 14-day readmission as outcome measure

\begin{tabular}{|c|c|c|c|}
\hline & & $\begin{array}{c}\text { 14-Day } \\
\text { Readmission } \\
\text { Odds Ratio }(95 \% \text { CI })\end{array}$ & $\mathbf{p}$ \\
\hline \multirow[t]{5}{*}{ Age } & $1-4$ & Reference & \\
\hline & $5-9$ & $0.8(0.3,1.9)$ & 0.598 \\
\hline & $10-14$ & $0.7(0.3,1.3)$ & 0.207 \\
\hline & $15-18$ & $0.8(0.4,1.6)$ & 0.567 \\
\hline & $19+$ & $1.4(0.5,3.6)$ & 0.494 \\
\hline \multirow[t]{2}{*}{ Decreased LV function } & Yes & $1(0.7,1.3)$ & 0.780 \\
\hline & No & Reference & \\
\hline \multirow[t]{2}{*}{ Arrhythmia } & Yes & $1.5(0.9,2.5)$ & 0.128 \\
\hline & No & Reference & \\
\hline \multirow[t]{2}{*}{ Respiratory support } & Yes & $0.6(0.3,1)$ & 0.045 \\
\hline & No & Reference & \\
\hline
\end{tabular}

